\title{
Aproximación al primer informe de ampliación para la comisión interamericana de derechos humanos - CIDH -: Gestión del riesgo de desastres naturales en Colombia como panorama general para el ejercicio de los derechos humanos en procesos de reasentamiento de población*
}

Approximation to the first report on extension for the inter-american commission on human rights (Interamerican Comission on Human Rights - IACHR: Managing the risk of natural disasters in Colombia as a general overview for the exercise of human rights in

processes of population resetting Jorge Eduardo Vásquez Santamaría ${ }^{\mathbf{x}}$ Martha Isabel Gómez Vélez ${ }^{2 \otimes}$ Hernán Darío Martínez Hincapié ${ }^{3}$

Fecha correspondencia:

Recibido: 1 de septiembre de 2017. Revisión: 6 de octubre de 2017. Aceptado: 1 de noviembre de 2017.

\section{Forma de citar:}

Vásquez, J.E., Gómez, M.

I., Martínez, H.D. (2017).

Aproximación al primer informe de ampliación para la comisión interamericana de derechos humanos - CIDH -: Gestión del riesgo de desastres naturales en Colombia como panorama general para el ejercicio de los derechos humanos en procesos de reasentamiento de población. Revista CES Derecho, (8), 2, julio - diciembre de 2017, 208-230.

\section{Open access}

Términos de uso

Licencia creative commons

Etica de publicaciones

Revisión por pares

\section{Resumen}

Como resultado de una Audiencia Temática celebrada ante la CIDH el 19 de marzo de 2015, la Clínica Jurídica de Interés Público de la Universidad Autónoma Latinoamericana (CJIP UNAULA) comparte la estructura del primer informe de ampliación y detalle de información, describiendo los segmentos y componentes principales a través de los cuales se busca acreditar el sustento que recomendó la CIDH cuando concluyó la Audiencia Temática con la finalidad de fortalecer las aseveraciones presentadas contra el Estado de Colombia. Una vez se hace la descripción general de los componentes del informe, se presentan de manera general algunos resultados concretos. Primero se aborda la gestión del riesgo de desastres como un derecho colectivo constitucionalmente reconocido en Colombia, seguidamente se da lugar a la evolución de la normativa sobre la gestión del riesgo como plataforma jurídica de la información ampliada sobre la realidad de los derechos humanos en poblaciones propensas a procesos de reasentamiento; y finalmente se da cuenta de los consolidados estadísticos departamentales sobre las víctimas del riesgo de desastres por eventos naturales en los últimos quince años.

Palabras clave: gestión del riesgo, derecho colectivo, reconstrucción normativa, desastre natural.

\section{Abstract}

As a result of a Thematic Hearing held before the IACHR on March 19, 2015, the Judicial Clinic of Public Interest of the Universidad Autónoma Latinoamericana (UNAULA) shares the structure of the first expansion report and information detail, describing the segments and Main components through 
Gestión por Open Journal

System

DOl: http://dx.doi.

org/10.21615/cesder.8.2.1

ISSN: 2145-7719

Sobre el artículo:

* Resultado final de la

investigación "Ejercicio de los

Derechos Humanos en procesos

de reasentamiento poblacional

a partir de la gestión del riesgo

de desastres por eventos

naturales en Colombia -

primera fase", ejecutado por

la Clínica Jurídica de Interés

Público de la Facultad de

Derecho de la Universidad

Autónoma Latinoamericana

en la línea de investigación

"Cultura Latinoamericana,

Estado y Derecho". Intervinieron

como auxiliares Karen

Viviana Rendón Osorio, Sara

Pulgarin Espinosa, Katherine

Bustamante González, María

Fernanda Barrera Cuartas, Ligni

Sigrid Ramírez Sánchez y Mateo

Palacio Sánchez.

Sobre los autores:

1. Abogado y Magíster en

Derecho de la Universidad de

Medellín. Ex coordinador de

la Clínica Jurídica de Interés

Público de la Universidad

Autónoma Latinoamericana.

2.Abogada y Magíster en

Derecho de la Universidad de

Antioquia. Coordinadora de

la Clínica Jurídica de Interés

Público de la Universidad

Autónoma Latinoamericana.

3.Abogado de la Universidad

Autónoma Latinoamericana

y Magíster en Derechos

Humanos de la Universidad

Sergio Arboleda. Docente

asesor de la Clínica Jurídica

de Interés Público de la

Universidad Autónoma

Latinoamericana. which it seeks to accredit the support it required and recommended the IACHR once the Thematic Hearing was concluded to strengthen the claims made against the State of Colombia. Once the general description of the components of the report is made, some concrete results are presented on the basis of disaster risk management as a collective right constitutionally recognized in Colombia; The evolution of regulations on risk management as a legal platform for expanded information on the reality of human rights in vulnerable populations prone to resettlement processes; And consolidated departmental statistics over the last fifteen years.

Key words: risk management, collective law, normative reconstruction, natural disaster.

\section{Introducción}

A partir de una Audiencia Temática ante la CIDH que tuvo lugar el 19 de marzo de 2015 como resultado del Informe de Petición sobre "Procesos de reasentamiento de población vulnerable en Colombia en el marco de procesos de reubicación de población desplazada, proyectos de desarrollo urbanístico, renovación urbana, y programas de prevención y atención del riesgo", resultado de un ejercicio de enseñanza clínica del Derecho realizado por la Alianza de Universidades por los Derechos Humanos, la Clínica Jurídica de la Corporación Universitaria Lasallista, y la CJIP UNAULA, se ejecutó el proyecto de investigación "Ejercicio de los Derechos Humanos en procesos de reasentamiento poblacional a partir de la gestión del riesgo de desastres por eventos naturales en Colombia", desde el cual se sustentan dos informes de ampliación y detalle de información para la $\mathrm{CIDH}$, requerimiento elevado por dicha Corporación en la Audiencia Temática.

El proyecto tiene por problema central ¿Cómo incide la gestión del riesgo de desastres por eventos naturales en Colombia en el ejercicio de los derechos humanos de las personas afectadas por procesos de reasentamiento poblacional? Para su desarrollo se proponen dos fases; la primera, ya finalizada y que sirve de base para el primer informe de ampliación, se construyó desde una retrospectiva sobre la gestión del riesgo de desastres por evento natural, toda vez que fuera de ser una de las variables propuestas a la CIDH en el Informe de Petición, es una materia legalmente imperativa para la planeación social y económica a través de los planes de desarrollo, y para la planeación territorial a través de los Planes de Ordenamiento Territorial, ambas obligaciones legales y reglamentarias a cargo de departamentos, municipios y distritos en Colombia.

En el Primer Informe se emprendió una reconstrucción jurídica e institucional que se posicionó como plataforma dogmática para la evaluación del contexto situacional que ha determinado el ejercicio de los Derechos Humanos en poblaciones propensas a programas y procesos de reasentamiento por la ocurrencia de eventos naturales riesgosos en un lapso de quince años (2000 a 2014) en las distintas regiones de Colombia. La citada reconstrucción se acompaña tanto de la reseña de casos emblemáticos decididos por Altas Corporaciones Judiciales como instancias de cierre jurisdiccional que han tenido la competencia para conocer y decidir situaciones directamente vinculadas a derechos comprometidos con la gestión del riesgo de desastres, la vivienda y el cumplimiento de la función pública del urbanismo, como por la reconstrucción de instrumentos internacionales que inciden de manera flexible pero creciente en el ordenamiento territorial a cargo de las entidades territoriales. Posteriormente el Primer Informe profundiza desde la organización política territorial gracias a la sistematización retrospectiva sobre las entidades departamentales en los últimos quince años en relación a los muertos, heridos, desparecidos, 
personas y familias registradas como víctimas provocadas por trece modalidades de evento natural, ofreciendo un consolidado estadístico nacional de atención de riesgos de desastres con base en la información recopilada por la Unidad Nacional de Gestión del Riesgo de Desastres (UNGRD) que a su vez recibe la información de la Defensa Civil colombiana, la Cruz Roja colombiana, el Sistema Nacional de Bomberos, los Comités Departamentales de Gestión de Riesgo de Desastres (CDGRD) y los Comités Municipales de Gestión de Riesgo de Desastres (CMGRD).

El Primer Informe finaliza con unas valoraciones que acentúan en el reconocimiento y protección de Derechos Humanos y la situación de derechos Sociales, Económicos, Culturales, Colectivos y del Ambiente; sobre los sistemas de información en la gestión del riesgo; la gestión del riesgo en la planeación y el ordenamiento territorial; la incorporación del riesgo de desastres como un determinante ambiental para los planes de desarrollo y los planes de ordenamiento territorial; la evolución normativa de la gestión del riesgo, y sobre los consolidados estadísticos departamentales en el periodo comprendido entre los años 2000 a 2014 en Colombia.

La retrospectiva sobre la gestión del riesgo de desastres se constituye no solo en el escenario que definió el ejercicio de los Derechos Humanos en los procesos de reasentamiento de población propensa a la ocurrencia de eventos naturales en un periodo delimitado entre los años 2000 a 2014, sino en la plataforma a partir de la cual se levanta la segunda fase, centrada en la proyección de las condiciones políticas que las autoridades competentes del Estado deben asegurar a través de instrumentos planificadores para el debido ejercicio de los Derechos Humanos. En esta oportunidad, la CJIP UNAULA presenta algunos de los resultados del Primer Informe de ampliación y detalle que desde la gestión del riesgo de desastres tienen una potencial incidencia en el ordenamiento territorial como uno de los marcos de garantía para los procesos de despojo, reasentamiento y reubicación poblacional.

\section{Breve referencia a la audiencia temática de la CIDH}

El proyecto de investigación que le dio origen a este escrito, surge gracias a la participación de la CJIP UNAULA como una de las peticionarias junto con la Alianza de Universidades por los Derechos Humanos y la Clínica Jurídica de la Corporación Universitaria Lasallista, para la realización de una audiencia temática ante la Comisión Interamericana de Derechos Humanos (CIDH), la cual tuvo lugar el 19 de marzo de 2015 en Washington y abordó el tema de "Procesos de reasentamiento de población vulnerable en Colombia en el marco de procesos de reubicación de población desplazada, proyectos de desarrollo urbanístico, renovación urbana, y programas de prevención y atención del riesgo".

En su participación como peticionaria de la audiencia temática, la CJIP UNAULA se incorporó con la elaboración global del Informe de Petición en el componente correspondiente con los procesos de reasentamiento poblacional originados por la ejecución de proyectos urbanísticos y de renovación urbana, así como en el rastreo y reseña de 20 casos emblemáticos nacionales judicializados donde se mostraban vulneraciones a diferentes personas y comunidades por alguna de las variables propuestas en el informe sobre procesos de reasentamiento de población vulnerable en Colombia.

Como uno de los resultados del informe de petición y de la audiencia temática, la CIDH manifestó la necesidad y conveniencia de hacer una ampliación y detalle de la información que sustentó las aseveraciones contra el Estado de Colombia. De ello 
que la CJIP UNAULA emprendiera la elaboración de dos informes de ampliación y detalle sobre aspectos de la situación de los Derechos Humanos de poblaciones propensas, aptas o relacionadas con procesos de reasentamientos a raíz de la ocurrencia de los eventos naturales que generan riesgo de desastre, emergencia o calamidad pública. En este escrito se hace referencia al primero de los informes abordando tres segmentos relevantes del mismo: La gestión del riesgo de desastres como un derecho colectivo constitucionalmente reconocido en Colombia, una aproximación a la evolución normativa nacional e internacional sobre la gestión del riesgo de desastres, y un acercamiento sobre los consolidados de víctimas en Colombia 2000 - 2014 y algunos precedentes de judicialización nacional para la protección de Derechos Humanos.

\section{La gestión del riesgo de desastres como un derecho colecti- vo constitucionalmente reconocido en Colombia}

Un resultado inicial radica en la naturaleza que la gestión del riesgo de desastres adquiere dentro del nuevo orden constitucional colombiano y los efectos que genera para el reconocimiento y protección de derechos. Si bien no figura como un derecho expresamente positivizado dentro del articulado de la Carta Superior, varias disposiciones si elevan dicha materia al rango constitucional. Es el caso del artículo 8 que da lugar al principio de corresponsabilidad cuando dispone que "Es obligación del Estado y de las personas proteger las riquezas culturales y naturales de la Nación", fuente de obligaciones para la gestión y manejo de los recursos ecosistémicos en un país diverso. En la misma dirección, y como una expresión de la especial responsabilidad ambiental que reviste al Estado, del artículo 80 se desprenden obligaciones como la de planificar el manejo y aprovechamiento de los recursos naturales para garantizar su desarrollo sostenible, su conservación, restauración o sustitución; prevenir y controlar los factores de deterioro ambiental, e imponer las sanciones legales y exigir la reparación de los daños causados al ambiente, estos últimos avalados también por el alcance del artículo 90. Además el artículo 215 prevé el Estado de Excepción por alteración del Orden Económico, Social y Ambiental, permitiendo intervenir en graves situaciones de desastres naturales o catástrofes humanas, y el artículo 339 exige incorporar en los Planes de Desarrollo las estrategias estatales en asuntos ambientales, y dentro de ellas, la gestión del riesgo, disposición que compromete al Sistema Nacional de Planeación con la gestión del riesgo del desastre, y a la luz de la legislación vigente, con la política nacional de gestión del riesgo de desastres y el Sistema Nacional de Gestión del Riesgo de Desastres (SNGRD).

En este sentido, la gestión del riesgo de desastres está lejos de ser una materia nueva e incipiente en el ordenamiento jurídico colombiano, y de allí, en el ejercicio de la función de los poderes públicos que actúan entorno a la misma, lo que acredita la necesaria preparación y actuación de las autoridades para garantizar el debido ejercicio de los derechos humanos involucrados en situaciones de riesgo, sean potenciales o consumadas, esto es, en la mitigación y prevención como en la reacción y atención.

Además, el reconocimiento constitucional de la gestión del riesgo de desastres no solo asegura una nueva dimensión del tratamiento de la materia a la luz del Estado Social de Derecho con el cumplimiento de las obligaciones a cargo del Estado para satisfacer las necesidades de los administrados, sino que postula la posibilidad de tratarse de una esfera de la actuación del Estado que por su desarrollo y madurez alcanzó los umbrales del reconocimiento constitucional a través del cual formalmente se garantizó el reconocimiento de un derecho que abarca un interés difuso o 
impersonal dentro de la sociedad, a la vez que está íntimamente correlacionado con otros bienes jurídicamente amparables, como el ambiente sano, el espacio público, la vivienda digna o el patrimonio público.

En desarrollo de los contenidos constitucionales, el ordenamiento legal y reglamentario abre una etapa en la evolución normativa sobre la gestión del riesgo de desastres, lo que no solo impulsa los avances normativos e institucionales sobre la materia, sino que contribuye a la formación de los contenidos y dimensiones de la gestión del riesgo como derecho colectivo. La Ley 472 de 1998 en el artículo 4, literal l, reconoce la prevención de desastres previsibles técnicamente como un derecho colectivo protegible a través de acción popular. Tan solo en este sentido, el reconocimiento como derecho colectivo según López, siguiendo a Kymlicka (2001, p. 27 a 29) hace de la gestión del riesgo de desastres un "derecho de un grupo a limitar las libertades de sus propios miembros en nombre de la solidaridad de un grupo o de la pureza cultural", o "al derecho de un grupo a limitar el poder político o económico ejercido sobre dicho grupo por la sociedad de la que forma parte con el objeto de asegurar que los recursos y las instituciones de que depende la minoría, no sean vulnerables a las decisiones de la mayoría". Por lo tanto, como derecho colectivo, la gestión del riesgo de desastres puede ser asumido como una meta colectiva, como la "particularización de la posición jurídica del colectivo" (Arango, 2005, p.84) determinante en las acciones positivas del Estado en procura de salvaguardar el interés general.

En ese orden, en Colombia la gestión del riesgo de desastres supera la dimensión de la función administrativa concentrada en el ejercicio de las competencias legales para la prestación del servicio público que demanda la prevención, mitigación, reacción y atención a las situaciones de riesgo, a una dimensión en la que como derecho colectivo se ampara en la obligación prestacional que tiene el Estado con el reconocimiento y la garantía de intereses difusos o colectivos, aunando además la garantía de protección judicial a través de una acción constitucional. Por lo tanto, la gestión del riesgo es entendida como una debida intervención del Estado dirigida a garantizar la protección y seguridad de todos sus habitantes, sea a nivel local, regional o nacional, con el fin de lograr la salvaguarda de unas condiciones mínimas de vida, de un bienestar social y de un desarrollo sostenible sobre todo en un contexto como el colombiano, en vía de desarrollo, con escenarios tan complejos, desiguales y socialmente diversos.

Para nuestro caso, siguiendo a Cardona (2009) la gestión del riesgo se plantea desde dos vías: de un lado, las causas y fenómenos que producen riesgos se deben a errores y confusiones en su previsión y entendimiento del territorio y los modelos de gestión, al no considerar la planeación territorial y la planeación del desarrollo consciente de estos fenómenos, igualmente las inapropiadas dinámicas de expansión urbana y la ausencia de compromiso técnico y político de los entes estatales para asumir la problemática, lo que implica, en definitiva, un riesgo para el derecho colectivo de la prevención y previsión de los desastres. De otra parte, es importante que las autoridades estatales encargadas de la gestión del riesgo y mitigación y adaptación al cambio climático tomen decisiones que prevean riesgos futuros y eviten inversiones de alto riesgo que puedan resultar en fenómenos de desastres naturales, lo que exige comprender y trabajar con experticia sobre las condiciones de vulnerabilidad, en la medida que la gestión adecuada constituye el contenido de la obligación clara y determinada dentro de la estructura y configuración de la gestión del riesgo como derecho colectivo que exige la estructuración de estrategias que 
atiendan las amenazas y eliminen la vulnerabilidad de forma eficiente y preventiva en favor de la comunidad en términos colectivos y difusos (Corte Constitucional, Sentencia T 041 de 2011).

En Colombia, el cumplimiento de esa función arrastra un sistema complejo de órganos, acciones, metodologías y conceptos para la prevención y atención de los desastres y eventos que ponen en riesgo los Derechos Humanos de los habitantes de zonas afectadas o propensas a eventos naturales o humanos catastróficos, lo que conduce a varios de los resultados obtenidos a partir de la reconstrucción normativa y su correspondiente valoración; en otras palabras, la gestión del riesgo de desastres como derecho colectivo de tipo prestacional reconocido constitucionalmente en Colombia, que acarrea el cumplimiento de una función administrativa a partir de un sistema y orientado por una política nacional, es en parte el resultado de la evolución de una plataforma jurídica e institucional para el ejercicio de los Derechos Humanos de poblaciones propensas a desastres naturales, plataforma que en la actualidad también es requerida para asegurar el amparo de los derechos y el cumplimiento de fines del Estado.

\section{Aproximación a la evolución normativa nacional e interna- cional sobre la gestión del riesgo de desastres}

La descripción de la evolución normativa e institucional sobre la gestión del riesgo de desastres como plataforma jurídica de la información ampliada sobre la realidad contextual para el ejercicio de los Derechos Humanos en poblaciones propensas a procesos de reasentamiento puede ser analizada diferenciando el contexto internacional del nacional. Desde el primero se abordaron dispositivos jurídicos con incidencia en el orden jurídico interno; primero se investigaron instrumentos internacionales contentivos de principios generales, como fue el caso de los principios rectores Deng y los principios rectores Pinheiro, luego se abordaron disposiciones sobre la gestión del riesgo como la Resolución 46/182 de la Asamblea General de la Organización de las Naciones Unidas; las disposiciones de la Conferencia Mundial sobre Reducción de los Desastres Naturales: Yokohama 1994; las disposiciones de la Cumbre Mundial sobre el Desarrollo Sostenible, la Declaración de Hyogo y el respectivo Marco de Acción de Hyogo: Aumento de la Resiliencia de las naciones y las comunidades ante el desastre; y el Marco de Acción de Sendai para la Reducción del Riesgo de Desastres.

Igualmente se ejecutó la descripción evolutiva del marco jurídico elaborado por la Comunidad Andina (CAN). En él tienen lugar el Programa Regional Andino para la Prevención y Mitigación de Riesgos y la Decisión 529 de 2002; la Decisión 555 de 2003: Convenio de financiación entre la Comunidad Europea y la Comunidad Andina para el proyecto de cooperación "Apoyo a la preparación y prevención de desastres en la Comunidad Andina"; la Decisión 591 de 2004: Estrategia Andina para la prevención y atención de Desastres; y la Decisión 713 de 2009: Aprobar la Estrategia Andina para la Prevención y Atención de Desastres - EAPAD. La normativa andina además condujo al estudio de la Convención Interamericana para facilitar la asistencia en casos de desastre, y de la Plataforma Regional para la reducción de Desastres de las Américas en sus cinco versiones.

Dentro de las disposiciones internacionales los principios rectores Deng y los principios rectores Pinheiro no son en sentido estricto figuras exclusivas encaminadas a la atención de poblaciones víctimas de desastres y catástrofes naturales, tampoco 
originadas a raíz de la desprotección de afectados por desastres naturales, pero si engloban dentro de sus contenidos axiológicos y jurídico políticos la protección de derechos Humanos de esa población.

Los principios Deng abarcan a los desplazados internos generados no solo por conflictos violentos o por violaciones graves a los Derechos Humanos, sino también los causados por eventos traumáticos, en los cuales se incluyen los eventos naturales provocadores de riesgo para el ejercicio de los Derechos Humanos. Incluir dichos principios en la reconstrucción jurídica e institucional para el ejercicio de los Derechos Humanos de poblaciones propensas o aptas a procesos de reasentamiento es necesario en la medida que desde ellos se fijen pautas para garantizar el reasentamiento y la reintegración en condiciones de seguridad. De lo anterior que los principios Deng reúnan entre las modalidades de desplazado interno a las personas que sin salir de las fronteras nacionales se han visto forzadas u obligadas a escapar o huir de su hogar o de su lugar de residencia habitual como consecuencia de catástrofes naturales. En el caso de los principios Pinheiro sus contenidos se dirigen a las personas que se hayan visto privadas de forma arbitraria o ilegal de sus anteriores hogares, tierras, bienes o lugares de residencia habitual, supuesto compatible con las situaciones tanto preventivas como de catástrofe generadas en procesos de gestión del riesgo del desastre en las que se busca asegurar posibles medidas de retorno.

La Resolución 44/236 de 1989 acogió el Marco Internacional de Acción para el Decenio Internacional para la Reducción de los Desastres Naturales, a partir del cual la Asamblea General vio la necesidad de mejorar la eficiencia de los esfuerzos colectivos internacionales en la prestación de asistencia humanitaria debido a la preocupación de la situación de las víctimas de desastres y situaciones de emergencia, de las cuales se desprenden pérdidas de vidas, desplazamientos y refugiados, y destrucción de bienes. La Resolución se divide en un conjunto de principios rectores, y disposiciones para la prevención, preparación, capacidad contingente, llamamientos unificados, coordinación, cooperación, liderazgo, y transición del socorro a la rehabilitación y el desarrollo.

Desde la Conferencia Mundial sobre Reducción de los Desastres Naturales: Yokohama 1994 resultaron un total de tres Resoluciones, de las cuales la número uno es de significativo valor, destinada a las directrices para la prevención de los desastres naturales, la preparación para casos de desastre y la mitigación de sus efectos, en que figuraban los principios, la estrategia y el plan de acción, condensadas todas en la denominada Estrategia de Yokohama.

En el año 2002 de la Declaración de Johannesburgo se desprendió el Plan de Acción sobre la protección y gestión de la base de recursos naturales del desarrollo económico y social, en el cual se reconoce el incremento de los impactos de las actividades del ser humano sobre los recursos naturales, lo cual promueve la degradación de los recursos, y exige estrategias que fortalezcan la capacidad para el aprovechamiento y protección de los recursos esenciales.

La importancia de los avances de la Conferencia de Yokohama de 1994, de la declaratoria del Decenio Internacional para la Reducción de los Desastres Naturales, y de la estrategia Internacional de Reducción de Desastres fue reconocida a partir de la Declaración de Hyogo. En la ocurrencia de los desastres se identifica un obstáculo para alcanzar el desarrollo sostenible y la erradicación de la pobreza, por lo que se reconoce la importancia de la cooperación, la solidaridad y las alianzas internacionales, así 
como la buena gobernanza (Hyogo, 2005, p.4). En Hyogo también se dispone la necesidad de establecer indicadores que permitan hacer seguimiento a las actividades de reducción del riesgo de los desastres que se ajusten a las circunstancias y capacidades particulares, posibilite la creación de mecanismos de intercambio de información sobre programas, iniciativas, prácticas, experiencias y tecnologías de apoyo, aspectos reunidos en el "Marco de Acción de Hyogo para 2005 - 2015: Aumento de la Resiliencia de las naciones y las comunidades ante los desastres".

Finalmente, del marco jurídico que ha venido siendo desarrollado por los Estados que integran la Comunidad Andina de Naciones (CAN) se identifican dos figuras concretas que develan destacados avances. La creación del Comité Andino para la Prevención y Atención de Desastres (CAPRADE) que encuentra entre sus fundamentos la reunión XI del Consejo Presidencial Andino en 1999 en Cartagena, donde se encargó de manera especial a la Corporación Andina de Fomento (CAF) ${ }^{1}$ de gestionar la cooperación necesaria para fortalecer y desarrollar las normas e instituciones para la prevención de riesgos, lo que dio lugar al Programa Regional Andino para la Prevención y Mitigación de Riesgos (PREANDINO). La labor encomendada a la CAF no era nueva. En 1998 la reunión de Presidentes de Guayaquil había encomendado la realización de un estudio sobre el impacto socioeconómico de los desastres a partir de la ocurrencia del fenómeno del niño.

Con la consolidación del PREANDINO se hacía necesario la creación de un organismo que asumiera la titularidad de las acciones que venían siendo previstas desde la CAN sobre la gestión del riesgo de desastres, especialmente a partir del PREANDINO. Lo anterior desencadenó la creación del CAPRADE a través de la Decisión 529 de 2002.

En lo que concierne al escenario nacional la evolución normativa puede ser dividida en tres momentos: 1970 a 1989, que inicia con el Código Nacional de Policía (Decreto 1355 de 1970 actualmente derogado por la Ley 1801 de 2016) y cierra con la Ley 46 de 1988, que le otorgó facultades al Presidente de la República para expedir normas que reformaran el título VIII de la Ley 9 de 1979, Código Nacional Sanitario. Resultado de dichas atribuciones se profirió el Decreto Ley 919 de 1989, que define el segundo momento en la evolución normativa nacional sobre la materia, para culminar con un último momento delimitado entre 1989 y 2012, año en el cual se promulga la Ley 1523 de 2012, que adopta la política nacional de gestión del riesgo de desastres y establece el SNGRD. No obstante, debe aclararse que por encima de la delimitación temporal han sido acogidas otras disposiciones que regulan componentes o dimensiones de la gestión del riesgo de desastres por eventos naturales; es el caso del Decreto Ley 019 de 2012, el Decreto 1807 de 2014, el Decreto 1077 de 2015 y el actual Código Nacional de Policía, Ley 1801 de 2016. El primero sujeta la revisión de los contenidos de mediano y largo plazo como la adopción de un nuevo POT, a que se acredite la delimitación y zonificación de las áreas de amenaza y en condiciones de riesgo, adicional a las medidas específicas para la mitigación. La labor de reglamentación que desde dicho artículo se desprendió para el Gobierno Nacional se cumplió a través del Decreto 1807 de 2014. En él se establecen las condiciones y escalas de detalle para incorporar de manera gradual la gestión del riesgo en la revisión de los contenidos de mediano y largo plazo en los POT (Artículo 1) restringiendo la presentación del proyecto de Acuerdo a la autoridad ambiental hasta que no se dé el lleno

1 Institución financiera constituida en 1970 que impulsa el desarrollo sostenible y la integración regional de América Latina mediante operaciones de crédito, recursos no reembolsables y apoyo en la estructuración técnica y financiera de proyectos de los sectores público y privado. (http://www. comunidadandina.org $/$ Seccion.. spx? $i d=50 \&$ tipo $=$ SA\&title=caf-banco-de-desarrollo-de-america-latina ) 
del requisito. Finalmente, el Decreto 1077 de 2015 compila la normativa vigente en materia de ordenamiento territorial en Colombia, incluyendo disposiciones sobre la gestión del riesgo de desastres desarrolladas por las normas citadas.

Al profundizar en la evolución de las normas reunidas en el primer momento histórico, se identifica que desde el Código de Policía no se establecieron instituciones permanentes destinadas a la atención de desastres, pues bien, el tratamiento de las mismas era potestad de funcionarios públicos determinados a quienes se les atribuía esta función con carácter de transitoriedad encargándose, antes que, de la gestión del riesgo, del manejo ambiental y de calamidades públicas. Con la Ley 9 de 1979 se tuvo un avance en lo que sería el desarrollo progresivo para llegar a lo que hoy se conoce como sistema integral de gestión de riesgo, pues, aunque vacila en el concepto de desastre, introduce las nociones de análisis de vulnerabilidad, medidas preventivas y planes de contingencia. Marca un avance significativo al involucrar a las entidades privadas prestadoras de servicios públicos en la realización del análisis de vulnerabilidad y al migrar de la asignación transitoria de funciones frente a la atención de desastres a la creación de un Comité Nacional de Emergencias para que ejerciera las mismas, que además debía reproducirse en los niveles regionales. De esta manera la Ley 9 de 1979 no solo institucionalizó la atención de emergencias, sino que estableció la descentralización de dicha institución, lo que a la postre conduciría a una mayor eficiencia y alcance en el desarrollo de sus actividades.

En 1984 el Decreto 1547 establece por primera vez un fondo común nacional cuya finalidad era proveer recursos con destinación exclusiva a la atención de necesidades surgidas en virtud de una situación de catástrofe. A diferencia de las normas anteriores, esta reglamentación se da a la tarea de realizar una definición del concepto de "catástrofe" que marcará su ámbito de aplicación material.

A diferencia de lo dispuesto por la Ley 9 de 1979 sobre la realización de planeamientos de operaciones de emergencia en cada circunscripción territorial, la Ley 46 de 1988 contempló la formulación de un Plan Nacional para la Prevención y Atención de Desastres que sirviera de derrotero y guía a las actuaciones regionales, al tiempo que le dio un mayor alcance a la intervención del sector privado respecto al tema de desastres, toda vez que amplío la realización del análisis de vulnerabilidad a las fases de prevención, manejo, rehabilitación, reconstrucción y desarrollo a que dan lugar las situaciones de desastre, e incluyó a la comunidad como sujeto de responsabilidades en dicho sistema.

El segundo momento histórico que define la normativa se concreta en el Decreto Ley 919 de 1989, marco reglamentario que rigió hasta el año 2012 cuando fue derogado por la Ley 1523. El Decreto 919 amplió y organizó el Sistema Nacional para la Prevención y Atención de Desastres creado por la Ley 46 de 1988, e introduce la obligación que tenían las circunscripciones territoriales de incluir dentro de sus planes de desarrollo un componente de prevención y atención de desastres. Además, establece la diferenciación entre desastre y calamidad, señalando como tal toda alteración de las condiciones normales de vida de la comunidad que no obstante no revestir la gravedad del desastre, es generada por las mismas causas. El Decreto 919 de 1989 también exigió a las dependencias de la administración central y descentralizadas incluir dentro de sus apropiaciones presupuestales las respectivas partidas para efectos de la prevención y atención de desastres.

Con la Constitución Política en 1991 se acoge un nuevo paradigma frente a la estructuración y funcionamiento del Estado para la realización de sus fines. En él, los 
Derechos Humanos de la población vulnerable por la gestión del riesgo se ven comprometidos en la totalidad del articulado. Basta exaltar que desde el preámbulo se fijó como cometido filosófico y axiológico del Estado colombiano "asegurar a sus integrantes la vida, la convivencia, el trabajo, la justicia, la igualdad, el conocimiento, la libertad y la paz, dentro de un marco jurídico, democrático y participativo que garantice un orden político, económico y social justo", el cual motiva la realización de los bienes jurídicos tutelables allí dispuestos, más aun, en poblaciones sujetas a condiciones de riesgo por desastres y emergencias naturales. Adicionalmente los derechos de las poblaciones vulnerables a los eventos naturales encuentran especial lugar en los fines del Estado Social de Derecho, en la medida que el artículo 2 dispone como tales: “(...) Las autoridades de la República están instituidas para proteger a todas las personas residentes en Colombia, en su vida, honra, bienes, creencias, y demás derechos y libertades, y para asegurar el cumplimiento de los deberes sociales del Estado y de los particulares."

Al amparo de la nueva Constitución el Decreto 969 de 1995 organizó y reglamentó la reserva para la atención de emergencias que tendría aplicación en el marco del SINPAD, previsto por la Ley 46 de 1988 y el Decreto 919 de 1989. Previó la existencia de la Red Nacional de Centros de Reserva para la atención de emergencias de conformidad con lo preceptuado en el artículo 65 del Decreto 919 de 1989, la cual tiene por propósito disponer de suministros básicos para las comunidades afectadas en cualquier departamento del país, así como facilitar los equipos y elementos especializados necesarios para la realización de actividades de control de eventos tanto naturales como antropogénicos y de rescate, búsqueda y salvamento con el fin de atender en forma adecuada y oportuna la primera respuesta frente a situaciones de emergencia que se presenten en el territorio nacional (Artículo 2).

La Red estaba organizada con centros de reserva regionales localizados en las treinta y dos capitales de departamento, así como en Bogotá, como instrumentos de apoyo logístico de los CREPAD, así como del Comité de Prevención y Atención de Desastres del Distrito Capital. Dos centros de reserva tenían carácter nacional y funcionarían en Bogotá bajo la administración de la Defensa Civil Colombiana y la Cruz Roja Colombiana, un tercero con fines de investigación estaba a cargo de la Universidad Nacional de Colombia, y los centros de respuesta inmediata estaban ubicados en todos los municipios y distritos del país y serían instrumento de apoyo logístico de los comités locales para la prevención y atención de desastres (Artículo 3). Esta disposición fue levemente modificada por el Decreto 3696 de 2009, al contemplar que la organización territorial estaría dada por los Centros de Reserva Regional localizados en los treinta y dos departamentos administrados por las entidades operativas del Sistema Nacional para la Prevención y Atención de Desastres; se mantienen los Centros de Reserva con carácter nacional funcionando desde Bogotá, y también los Centros de Respuesta Inmediata ubicados en los municipios, por lo que desaparece el centro con fines de investigación a cargo de la Universidad Nacional de Colombia.

Como lo previó el Decreto 919 de 1989, se mantiene vigente que en la elaboración de los planes de desarrollo las autoridades tendrán en cuenta las apropiaciones presupuestales para el sostenimiento y fortalecimiento de los centros de reserva y los centros de respuesta inmediata para la atención de emergencias (Artículo 7). La norma también indicaba el procedimiento para la transferencia de bienes donados a la Red por intermedio del Fondo Nacional de Calamidades, disposición que fue modificada por el Decreto 3696 de 2009 señalando que el "Fondo Nacional de Ca- 
lamidades, transferirá, a título gratuito, mediante acto administrativo motivado, los bienes que adquiera o reciba en donación el citado Fondo, para el fortalecimiento y operación de los Centros de Reserva, a las entidades operativas del Sistema Nacional para la Prevención y Atención de Desastres, los cuales deberán ser utilizados en actividades inherentes a la prevención y atención de emergencias en el territorio nacional."

El Decreto $2190^{2}$ también fue expedido en el año 1995, y con él se ordena la elaboración y desarrollo del Plan Nacional de Contingencia contra derrames de Hidrocarburos, Derivados y Sustancias Nocivas en aguas marinas, fluviales y lacustres como instrumento rector del diseño y realización de actividades dirigidas a prevenir, mitigar y corregir los daños que estos puedan ocasionar (artículo 1), para lo cual dispone la creación del Comité Técnico para la elaboración del mismo.

En 1996 se expide la Ley 322 que crea el Sistema Nacional de Bomberos, la cual fue derogada por la Ley 1575 de 2012. La Ley 322 contempló que la prevención de incendios era responsabilidad de todas las autoridades y habitantes del territorio nacional, por lo que se debía contemplar la contingencia de este riesgo en los bienes inmuebles, programas de desarrollo urbanístico e instalaciones y adelantar planes, programas y proyectos tendientes a disminuir su vulnerabilidad (Artículo 1). Además, el artículo 4 dispuso que el Sistema Nacional de Bomberos de Colombia formaba parte del SINPAD creado por la Ley 46 de 1988 y reglamentado por el Decreto 919 de 1989, y creó el Fondo Nacional de Bomberos como subcuenta del Fondo Nacional de Calamidades (Artículo 5). En armonía con lo anterior, el artículo 6 preceptuó que los Cuerpos de Bomberos; las Delegaciones Departamentales de Bomberos y la Delegación Distrital de Santa Fe de Bogotá; la Dirección Nacional para la Atención y Prevención de Desastres del Ministerio del Interior; la Junta Nacional de Cuerpos de Bomberos de Colombia; y la Delegación Nacional de Bomberos, integraban el Sistema Nacional de Bomberos.

La Ley 388 de 1997 señala como sus objetivos (Artículo 1, numerales 2 y 3) el establecimiento de mecanismos que permitan al municipio la prevención de desastres en asentamientos de alto riesgo, velar por la protección del medio ambiente y la prevención de desastres respecto de la utilización del suelo por parte de sus propietarios. La norma establece que el ordenamiento territorial es una función pública que pretende alcanzar, entre otros, una mayor seguridad de los asentamientos humanos ante los riesgos naturales (artículo 3, numeral 4). Como modalidades de acciones urbanísticas contempla determinación de las zonas no urbanizables que presenten riesgos para la localización de asentamientos humanos, por amenazas naturales, o que de otra forma presenten condiciones insalubres para la vivienda (artículo 8 , numeral 5) y localizar las áreas críticas de recuperación y control para la prevención de desastres (artículo 8, numeral 11).

En el artículo 10 señala que la conservación y protección del medio ambiente, los recursos naturales, la prevención de amenazas y riesgos naturales constituyen uno de los elementos determinantes que se deberán tener en cuenta para la elaboración y adopción del Plan de Ordenamiento Territorial (POT), el cual deberá contener dentro de su componente general la determinación y ubicación en planos de las zonas que presenten alto riesgo para la localización de asentamientos humanos,

2. El Plan Nacional de Contingencia contra Derrames de Hidrocarburos, derivado y Sustancias Nocivas en aguas marinas, fluviales y lacustres -PNC-, fue adoptado mediante el Decreto 321 de 1999, el cual contiene los objetivos generales y específicos, los principios generales que lo guiarán, las facultades que tendrá la Dirección Nacional para la Prevención y Atención de Desastres en el marco de este plan, su estructura (plan estratégico, plan Operativo y plan Informativo), su incorporación en los planes de contingencias de quienes tengan manejo de hidrocarburos, derivados o sustancias nocivas, la obtención de recursos para su implementación y las disposiciones relativas a el seguimiento y evaluación de la ejecución del PNC. 
por amenazas, riesgos naturales o condiciones de insalubridad (artículo 12, numeral 2.3). Además, los POT dentro de su componente urbano deberán tener en cuenta la delimitación de áreas expuestas a amenazas y riesgos naturales y el establecimiento de mecanismos para la reubicación de los asentamientos humanos localizados en zonas de alto riesgo donde se incluyan estrategias para evitar su nueva ocupación. (Artículo 13, numerales 3 y 5). Respecto al componente rural del POT se establece como mínimo prever, entre otros, la delimitación de las áreas de conservación y protección de los recursos naturales paisajísticos, geográficos y ambientales, incluyendo las áreas de amenazas y riesgos, o que formen parte de los sistemas de provisión de los servicios públicos domiciliarios o de disposición final de desechos sólidos o líquidos (Artículo 14, numeral 3) contenidos que deberán ser tenidos en cuenta también en los planes básicos de ordenamiento territorial en cada uno de sus componentes según lo preceptuado en el artículo 16 de la Ley 388 de 1997, y por mandato del artículo 17, los esquemas de ordenamiento territorial, que deberán contener como mínimo los objetivos, estrategias y políticas a largo y mediano plazo para la determinación de las zonas de amenazas y riesgos naturales y las respectivas medidas de protección.

Como justificación de utilidad pública para motivar acto administrativo expropiatorio, el artículo 58 (literales b y m) establece la reubicación de asentamientos humanos ubicados en sectores de alto riesgo y el traslado de las poblaciones por riesgos físicos inminentes, y, adicionalmente establece sanciones para quienes parcelen, urbanicen o construyan en zonas calificadas como de riesgo. (Artículo 104, numeral 1)

Finalmente, la Ley 388 prevé en el artículo 35 la categorización de suelo de protección para las áreas de amenazas y riesgos no mitigable donde existan asentamientos humanos, las cuales tienen restringida la posibilidad de urbanizarse; además, como tratamiento de las áreas catalogadas como de riesgo no recuperable que hayan sido desalojadas, prevé la entrega de las mismas a las Corporaciones Autónomas Regionales o la autoridad ambiental de la respectiva circunscripción territorial para su manejo y prevención de una nueva ocupación. Dicho desalojo deberá efectuarse mediante planes o proyectos de reubicación de asentamientos humanos (artículo 121), lo que una vez más pone de manifiesto la necesidad de contar de manera previa con reglamentaciones específicas para procesos de desalojo y reubicación para trasferir los suelos a las autoridades señaladas.

En el año 1998 se expidió el Decreto 93, por el cual se adopta el Plan Nacional para la Prevención y Atención de Desastres ${ }^{3}$. El Plan tiene como objetivo orientar el accionar civil y estatal respecto a la prevención y mitigación del riesgo y los preparativos en caso de desastre, contribuyendo de esta forma no sólo a reducción del riesgo sino también al desarrollo sostenible de las comunidades vulnerables. Adicionalmente insta a anexar al Plan Nacional el documento "Fundamentos y Acción Programática del Plan Nacional para la Prevención y Atención de Desastres", el cual sería elaborado por la Dirección Nacional y sometido a consideración del Comité Nacional para su aprobación, fija los objetivos, estrategias y programas a seguir, entre los cuales sobresale los relacionados con la incorporación de la prevención y reducción de riesgos en la planificación, donde la norma reúne la incorporación de criterios preventivos y de seguridad en los planes de desarrollo, el manejo y tratamiento de asentamientos humanos y de infraestructura localizados en zona de riesgo, y la articulación de la política ambiental y de prevención de desastres. En este punto, y antes de citar los programas de fortalecimiento

3. Por medio del documento CONPES 3146 de 2001 se establecen estrategias para consolidar la ejecución del Plan Nacional para la Prevención y Atención de Desastres. Dichas estrategias consisten principalmente en un "conjunto de acciones prioritarias para mejorar el desarrollo de las cuatro líneas programáticas del PNPAD, en cuanto a: 1) el conocimiento; 2) la incorporación del tema en la planificación; 3) el fortalecimiento institucional del SNPAD; y 4) el mejoramiento de los programas de educación y divulgación. Igualmente, incluye aspectos de orden financiero y de seguimiento, necesarios para consolidar el PNPAD". 
del Desarrollo Institucional, es obligado exaltar para el caso que convoca el informe, que los programas relacionados con la planificación están directamente vinculados a la obligación del Estado de elaborar instrumentos, metodologías y normas a la luz del plan de desarrollo con la finalidad de reducir el riesgo y asignar recursos para la prevención y atención de desastres. Igualmente se identifica que, dentro del manejo y tratamiento de asentamientos humanos, la reglamentación exige para el municipio la elaboración de inventarios de viviendas en riesgo, impulsar programas de reubicación, mejoramiento y protección de vivienda, promocionando la reglamentación de usos del suelo y el ordenamiento territorial con fines preventivos. Además, debe el municipio:

Elaborar normas de seguridad y reglamentos de diseño y construcción de edificaciones e infraestructura de líneas vitales, intervenir y reducir la vulnerabilidad de centros urbanos, edificaciones indispensables e infraestructura de líneas vitales existentes y estudiar y promover la aplicación de seguros para la protección de los bienes y servicios individuales y colectivos (Decreto 93, 1998: artículo 7).

Para el 2012, se promulga la Ley 1523 que adopta la política nacional de gestión del riesgo de desastres y se establece el Sistema Nacional de Gestión del Riesgo de Desastres, y con ello, introduce de manera expedita un conjunto de disposiciones que articulan y armonizan la gestión del riesgo de desastres con el ordenamiento territorial y la planeación económica y social. Es así como entre sus artículos 38 a 42 dispone la incorporación de la gestión del riesgo en la inversión pública, la integración de la gestión del riesgo en la planificación territorial y del desarrollo, la incorporación en la planificación, la incidencia en la planificación del desarrollo y el ordenamiento territorial, y el análisis específico de riesgos y la elaboración de planes de contingencia.

Entre las disposiciones jurídicas vigentes del ordenamiento jurídico nacional se destacan el Decreto Ley 019 de 2012, el Decreto 1807 de 2014 y el Decreto 1077 de 2015. El primero de ellos sujeta tanto la revisión de los contenidos de mediano y largo plazo, como la adopción de un nuevo plan de ordenamiento territorial, a que se acredite la delimitación y zonificación de las áreas de amenaza y en condiciones de riesgo, adicional a las medidas específicas para la mitigación. La labor de reglamentación que desde dicho artículo se desprendió para el Gobierno Nacional, se cumplió a través del Decreto 1807 de 2014. En él se establecen las condiciones y escalas de detalle para incorporar de manera gradual la gestión del riesgo en la revisión de los contenidos de mediano y largo plazo en los POT (Artículo 1) restringiendo la presentación del proyecto de Acuerdo a la autoridad ambiental hasta que no se del lleno del citado requisito. No obstante, llama la atención que la norma reglamentaria se restrinja a los eventos de movimiento en masa (Artículo 8) inundación (Artículo 9) y avenida torrencial (Artículo 10) cuando la UNGRD sistematiza información sobre más de trece modalidades de evento natural. Finalmente, el Decreto 1077 de 2015 compila la normativa vigente en materia de ordenamiento territorial en Colombia, incluyendo disposiciones sobre la gestión del riesgo de desastres.

\section{Sobre los consolidados de víctimas en Colombia 2000 - 2014 y al- gunos precedentes de judicialización nacional para la protección de Derechos Humanos.}

La evolución normativa sobre la Gestión del Riesgo de Desastres de la que ha sido objeto el ordenamiento jurídico no se compagina con los resultados de víctimas recolectados en el acontecer nacional año tras año. El fortalecimiento y modernización del sistema nacional para desplegar la función administrativa de la gestión del riesgo de 
desastres, fuertemente respaldado en las disposiciones internacionales, además por la descentralización, la planeación socioeconómica y territorial, y la corresponsabilidad entre entidades públicas, privadas y comunitarias, aun permanece alejado de la posibilidad de ser valorado como un sistema preventivo en el cual el ejercicio de los Derechos Humanos no sean objeto de medidas reactivas y paliativas, sino resultados de la planeación, de medidas preventivas, precautorias y de efectiva mitigación.

A partir de la sistematización y consecuente consolidación del número de muertos, heridos, desaparecidos, personas, y familias registradas a causa de trece (13) eventos naturales: avalanchas, contaminación, deslizamiento, erosión, granizadas, heladas, inundaciones, marejadas, sequias, sismos, tormentas eléctricas, vendavales y erupciones volcánicas ante la UNGRD en los treinta y dos (32) departamentos de Colombia y el Distrito Capital, se identifica una serie de fallas frente a la gestión del riesgo de desastres por eventos naturales, lo que afecta el cumplimiento de la gestión como función administrativa, y con ello, se debilitan las acciones para asegurar la efectiva realización de la prevención de desastres técnicamente previsibles y ejercer debidamente otros Derechos Humanos correlacionales.

Lo anterior se soporta no solo en el número de repeticiones de los eventos naturales, los cuales en el período que comprende los años 2007 a 2012 aumentaron tendencialmente, sino en que a partir de su repetición histórica y el incremento de muchos de ellos en zonas determinadas del territorio, las cifras de muertos, heridos, desaparecidos, personas, y familias registradas evidencia una constante que no decrece, y por el contrario, parece fijar una base de afectación sobre la cual se desenvuelve la gestión del riesgo de desastres. En otras palabras, las cifras dramáticas de muertos, heridos, desaparecidos, personas, y familias registradas en periodos como los comprendidos entre los años 2010 a 2012 en los que se identifican picos o incrementos de afectaciones por la intensidad o recurrencia de los eventos naturales, resultan tener como polo opuesto una base de afectación que se consolida a partir de un número no escandaloso de víctimas. Así se devela de la sistematización de las cifras depositadas en la UNGRD, en la cual el mayor número de personas que perdieron la vida a raíz de eventos hidrometeorológicos pone en evidencia la tendencia al no decrecimiento desde eventos como las avalanchas, el deslizamiento y las inundaciones, reafirmando períodos críticos consecutivos para el ejercicio de Derechos Humanos como los vividos entre los años 2000 - 2002 y los años 2007 - 2012.

\begin{tabular}{|c|c|c|c|c|c|c|c|c|c|c|c|c|c|c|c|}
\hline \multirow[b]{2}{*}{ Fenómemo } & \multicolumn{14}{|c|}{ Total Nacional de número de muertos por eventos Hidrometeorológicos 2000 - 2014} & \multirow[b]{2}{*}{2014} \\
\hline & 2000 & 2001 & 2002 & 2003 & 2004 & 2005 & 2006 & 2007 & 2008 & 2009 & 2010 & 2011 & 2012 & 2013 & \\
\hline Avalancha & 0 & 0 & 7 & 11 & 0 & 57 & 12 & 10 & 15 & 10 & 25 & 8004 & 261 & 0 & 7 \\
\hline Deslizamiento & 48 & 57 & 20 & 49 & 41 & 90 & 129 & 59 & 140 & 47 & 141 & 145 & 56 & 36 & 33 \\
\hline Inundación & 29 & 6 & 12 & 42 & 13 & 76 & 18 & 32 & 24 & 34 & 72 & 118 & 22 & 19 & 13 \\
\hline $\begin{array}{l}\text { Tormenta } \\
\text { eléctrica }\end{array}$ & 0 & 0 & 0 & 0 & 0 & 0 & 8 & 1 & 1 & 2 & 14 & 14 & 23 & 18 & 0 \\
\hline Vendaval & 0 & 2 & 4 & 1 & 2 & 65 & 8 & 6 & 4 & 1 & 5 & 9 & 6 & 4 & 97 \\
\hline Total anual & 77 & 65 & 43 & 103 & 56 & 288 & 175 & 108 & 184 & 94 & 257 & 8290 & 368 & 77 & 150 \\
\hline
\end{tabular}

Fuente: Vásquez, J. E., Gómez, M. I. \& Martínez, H. D. (2017) Gestión del riesgo de desastres, ordenamiento territorial, reasentamiento y reubicación: correlación desequilibrada para el ejercicio de derechos humanos desde retrospectivas jurídicas en Colombia. En: IX Seminario Internacional de Investigación en Urbanismo. Barcelona.

Los consolidados cronológicos derivados de los eventos naturales hidrometeorológicos refuerzan la premisa no solo sobre las modalidades de los eventos naturales que se constituyen como principales causas de afectación a las poblaciones, sino del desbordamiento en el número de afectados por encima de la capacidad preventiva 
e incluso reactiva de las autoridades competentes, en la medida que la tendencia entre los años 2007 - 2012 da cuenta de una ocurrencia sostenida en el tiempo, intensificada en el pico $2010-2011$.

Igualmente se confirma la perjudicial incidencia de los eventos de avalancha, deslizamiento, inundación y vendaval en los consolidados correspondientes con el número de familias registradas como afectadas por los eventos naturales. En ellos se hace mas evidente y detallada no solo la tipología del evento catastrófico sino la tendencia histórica de la afectación, toda vez que a diferencia de los resultados sobre heridos y muertos en el periodo 2007 - 2012, las cifras de familias afectadas por la gestión del riesgo de desastres ponen en evidencia la crítica situación enfrentada entre los años 2000 - 2002.

\begin{tabular}{|c|c|c|c|c|c|c|c|c|c|c|c|c|c|c|c|}
\hline \multicolumn{16}{|c|}{ Total Nacional de heridos por eventos hidrometeorológicos 2000 - 2014} \\
\hline Fenómeno & 2000 & 2001 & 2002 & 2003 & 2004 & 2005 & 2006 & 2007 & 2008 & 2009 & 2010 & 2011 & 2012 & 2013 & 2014 \\
\hline Avalancha & 0 & 0 & 0 & 15 & 7 & 80 & 10 & 1 & 6 & 15 & 5 & 8003 & 194 & 1 & 1 \\
\hline Deslizamiento & 71 & 34 & 16 & 65 & 70 & 101 & 129 & 76 & 170 & 34 & 182 & 150 & 72 & 87 & 17 \\
\hline Tormentas eléctrica & 0 & 0 & 0 & 0 & 0 & 0 & 66 & 5 & 0 & 3 & 67 & 0 & 33 & 31 & 0 \\
\hline Vendaval & 18 & 225 & 3 & 5 & 16 & 0 & 112 & 28 & 34 & 31 & 30 & 13 & 194 & 52 & 374 \\
\hline Total anual & 89 & 259 & 19 & 85 & 93 & 181 & 317 & 110 & 210 & 83 & 284 & 8166 & 493 & 171 & 392 \\
\hline
\end{tabular}

Fuente: Vásquez, J. E., Gómez, M. I. \& Martínez, H. D. (2017) Gestión del riesgo de desastres, ordenamiento territorial, reasentamiento y reubicación: correlación desequilibrada para el ejercicio de derechos humanos desde retrospectivas jurídicas en Colombia. En: IX Seminario Internacional de Investigación en Urbanismo. Barcelona.

\begin{tabular}{|c|c|c|c|c|c|c|c|c|c|c|c|c|c|}
\hline \multirow[b]{2}{*}{ Fenómeno } & \multicolumn{12}{|c|}{ Total nacional de familias registradas por eventos hidrometeorológicos 200 - 2012} & \multirow[b]{2}{*}{2012} \\
\hline & 2000 & 2001 & 2002 & 2003 & 2004 & 2005 & 2006 & 2007 & 2008 & 2009 & 2010 & 2011 & \\
\hline Avalancha & 437 & 21 & 1165 & 583 & 2427 & 4858 & 2158 & 830 & 2665 & 1503 & 23241 & 8006 & 3154 \\
\hline Contaminación & 1204 & 0 & 0 & 5 & 0 & 0 & 641 & 9 & 0 & 4 & 0 & 0 & 0 \\
\hline Deslizamiento & 5598 & 810 & 3427 & 1394 & 8479 & 11620 & 9732 & 3873 & 14661 & 8378 & 18949 & 9 & 11527 \\
\hline Erosión & 0 & 0 & 0 & 0 & 583 & 294 & 0 & 1557 & 0 & 154 & 691 & 0 & 77 \\
\hline Erupción & 0 & 0 & 0 & 30 & 0 & 0 & 0 & 973 & 1014 & 0 & 0 & & 5 \\
\hline Granizada & 0 & 0 & 268 & 43 & 0 & 0 & 491 & 230 & 7 & 3 & 208 & 320 & 435 \\
\hline Helada & 0 & 0 & 0 & 0 & 3000 & 0 & 0 & 0 & 0 & 0 & 1228 & 0 & 0 \\
\hline $\begin{array}{l}\text { Incendio } \\
\text { forestal }\end{array}$ & 9 & 4990 & 111 & 83 & 53 & 89 & & 0 & 0 & 80 & 31 & 0 & 4990 \\
\hline Inuncación & 81616 & 19181 & 45685 & 67034 & 123846 & 20368 & 114353 & 290657 & 344292 & 57644 & 563364 & 0 & 123903 \\
\hline Marejada & 0 & 0 & 0 & 0 & 0 & 0 & 1928 & 1928 & & 0 & 0 & 0 & 86 \\
\hline Sismo & 97 & 0 & 0 & 100 & 2786 & 227 & 0 & 2482 & 4386 & 27 & 33 & 3 & 1849 \\
\hline $\begin{array}{l}\text { Tormenta } \\
\text { aléctrica }\end{array}$ & 0 & 0 & 0 & 0 & 0 & 0 & 0 & 0 & 0 & 1 & 15 & 82 & 149 \\
\hline Vendaval & 4312 & 13973 & 8021 & 11151 & 17918 & 171789 & 19293 & 14273 & 12892 & 17493 & 20410 & 34732 & 585599 \\
\hline Sequía & 0 & 1609 & 6480 & 6251 & 8726 & 2376 & 300 & 4100 & 0 & 2463 & 382783 & & 5897 \\
\hline Total anual & 93273 & 40584 & 58677 & 80423 & 167818 & 209245 & 148596 & 316812 & 24610 & 85287 & 20027 & 43152 & 731774 \\
\hline
\end{tabular}

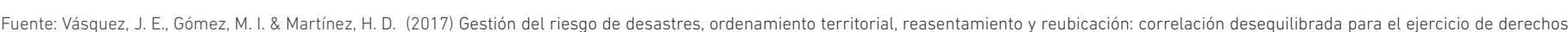
humanos desde retrospectivas jurídicas en Colombia. En: IX Seminario Internacional de Investigación en Urbanismo. Barcelona.

Esta primera realidad que ofrecen los consolidados estadísticos conduce a postular que la gestión del riesgo de desastres en Colombia aun carece de eficacia en su dimensión de planeación y prevención y se desenvuelve primordialmente en términos reactivos que no han logrado dar cuenta de la disminución de afectación de derechos en varios años consecutivos. Si bien puede resultar imposible llevar a niveles cero las cifras de afectación de bienes jurídicos como la vida, la integridad personal, la seguridad y la familia, generando como base de la gestión del riesgo de desastres la no afectación de derechos en el territorio, la tradicional tendencia histórica en la ocurrencia, 
intensidad y localización de los eventos naturales deja ver las fallas de la función administrativa de la gestión del riesgo de desastres, y de todos los principios que guían dicha función a un nivel de una aparente tolerancia de perdidas humanas y naturales que se consolidan en un umbral de ejercicio de la gestión del riesgo de desastres.

Incluso la tendencia cronológica permite postular que las víctimas por la ocurrencia, intensidad y localización de muchos de los eventos naturales se presenta a raíz de fenómenos que alteran o intensifican las condiciones de los eventos naturales tradicionales del territorio nacional, como los ocurridos periódicamente en el océano pacífico. Sobre esos fenómenos ambientales se tiene conocimiento previo que permite desplegar acciones preventivas e incluso precautorias que lleven a medidas prohibitivas para proteger Derechos Humanos. No obstante, los consolidados nacionales ponen al descubierto que los fenómenos naturales oceánicos o de dimensiones continentales de los cuales se tiene previo conocimiento, al contrario de posibilitar la disminución de las cifras de afectados, son la causa del incremento exponencial de muertos, heridos, desaparecidos, personas, y familias registradas en territorios claramente catalogados como vulnerables. Cabe anotar que esos eventos que pudieron ser prevenidos y mitigados desde el factor de previsibilidad y análisis de vulnerabilidad sin la influencia o incidencia de fenómenos oceánicos como el fenómeno de la niña o el niño, son generadores preocupantes de afectaciones a Derechos Humanos que se mantienen en el SNGRD, y bajo la presencia de fenómenos oceánicos antes de reflejar el decrecimiento de afectaciones por el ejercicio preventivo frente a su ocurrencia, permiten incrementos.

Además es claro que los consolidados dan cuenta que eventos como la avalancha, el deslizamiento y la inundación (de carácter hidrometeorológico) son los que más afectan el territorio nacional y son los más desastrosos en cuanto a la afectación a población en condiciones de vulnerabilidad en contravía con los eventos que han sido atendidos de manera principal por la institucionalidad y la sociedad en general como son sismo y erupción, muestra de ello son las cifras que arroja el período de 2010-2011, años durante los cuales se da la denominada "Ola Invernal", y frente a la cual el Estado, antes que prevenir o hacer esfuerzos para mitigar, termina reaccionando cuando el desastre o la catástrofe ya se ha presentado, a pesar de su previsibilidad por la dinámica de sus condiciones en años anteriores tales como 2006 y 2007 respectivamente. En el siguiente cuadro se puede ver como en 2010 los eventos naturales que más se presentaron están relacionados con esas condiciones hidrometeorológicas del país atendiendo a su ubicación geográfica, entre otros aspectos.

La ocurrencia, intensidad y localización de los eventos, sumado a la vulneración de Derechos Humanos en las poblaciones de esos territorios, proporciona desde la tendencia histórica, la certeza que los eventos naturales hidrometeorológicos son la fuente de mayor ocurrencia de desastres y la principal causa de vulneración a la tutela del ejercicio de los Derechos Humanos. Si bien se identifica un período crítico comprendido entre los años 2010 - 2011 "el número de repeticiones de diversos eventos naturales en el período que comprende los años 2007 - 2012 aumenta tendencialmente y no presenta variaciones significativas de disminución, lo que hace de los eventos procesos que pudieron ser prevenidos y mitigados desde el factor de previsibilidad y análisis de vulnerabilidad" (Vásquez, Gómez y Martínez, 2017, p. 11). Lo anterior puede ser detallado a partir de los consolidados departamentales cronológicos sobre el evento de deslizamiento e inundación. 


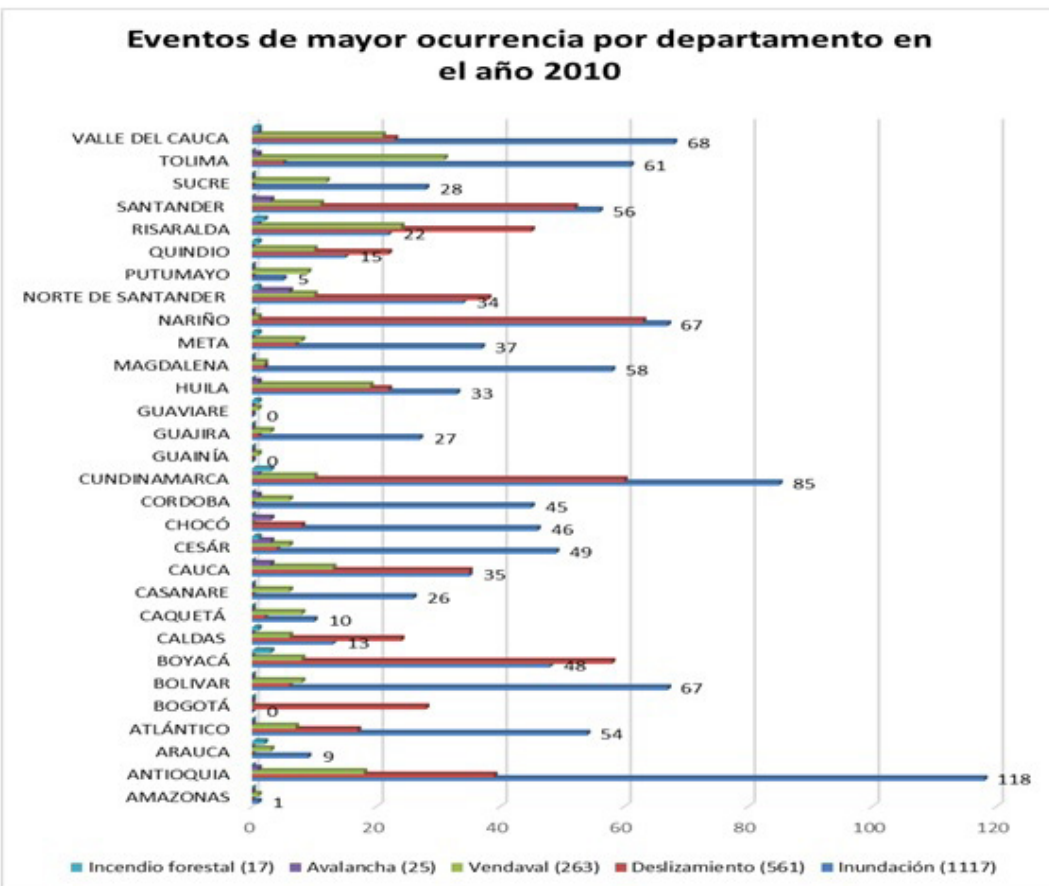

Elaboración propia de los autores a partir de los datos recolectados por la Unidad Nacional de Gestión del Riesgo de Desastres - UNGRD a través del Sistema de Información para la Gestión del Riesgo de Desastres, en su componente Consolidados atención de emergencias, conforme lo dispone el numeral 10 del artículo 4 del decreto 4147 de 2011

\begin{tabular}{|c|c|c|c|c|c|c|c|c|c|c|c|c|c|c|c|}
\hline \multicolumn{16}{|c|}{ Frecuencia de evento natural inundación descriminado por departamentos entre 2000 - 2014} \\
\hline Departamento & 2000 & 2001 & 2002 & 2003 & 2004 & 2005 & 2006 & 2007 & 2008 & 2009 & 2010 & 2011 & 2012 & 2013 & 2014 \\
\hline Amazonas & 8 & 2 & 8 & 3 & 2 & 0 & 1 & 1 & 2 & 5 & 1 & 3 & 2 & 0 & 1 \\
\hline Antioquia & 23 & 11 & 10 & 20 & 19 & 43 & 36 & 46 & 95 & 62 & 118 & 160 & 56 & 58 & 27 \\
\hline Arauca & 4 & 1 & 6 & 5 & 7 & 2 & 19 & 5 & 6 & 11 & 9 & 15 & 24 & 9 & 8 \\
\hline Atlántico & 10 & 3 & 5 & 7 & 19 & 27 & 14 & 40 & 46 & 10 & 54 & 56 & 37 & 22 & 6 \\
\hline Bolívar & 1 & 1 & 15 & 26 & 55 & 44 & 6 & 66 & 78 & 6 & 67 & 72 & 23 & 12 & 15 \\
\hline Boyacá & 2 & 0 & 4 & 15 & 9 & 10 & 36 & 5 & 43 & 11 & 48 & 3 & 82 & 18 & 8 \\
\hline Caldas & 1 & 0 & 1 & 2 & 5 & 6 & 4 & 5 & 13 & 0 & 13 & 22 & 27 & 12 & 6 \\
\hline Caquetá & 4 & 3 & 1 & 2 & 5 & 3 & 1 & 8 & 2 & 9 & 10 & 21 & 17 & 18 & 15 \\
\hline Casanare & 3 & 0 & 17 & 11 & 13 & 22 & 22 & 5 & 16 & 12 & 26 & 23 & 46 & 18 & 11 \\
\hline Cauca & 5 & 1 & 7 & 2 & 16 & 12 & 14 & 13 & 81 & 24 & 35 & 74 & 55 & 22 & 29 \\
\hline Cesar & 32 & 6 & 2 & 8 & 19 & 20 & 20 & 45 & 42 & 12 & 48 & 62 & 32 & 17 & 13 \\
\hline Chocó & 18 & 15 & 27 & 35 & 15 & 36 & 8 & 29 & 39 & 24 & 46 & 71 & 68 & 38 & 24 \\
\hline Córdoba & 3 & 4 & 3 & 25 & 31 & 9 & 21 & 42 & 39 & 26 & 45 & 42 & 50 & 22 & 11 \\
\hline Cundinamarca & 4 & 4 & 11 & 10 & 17 & 23 & 59 & 18 & 109 & 33 & 85 & 242 & 122 & 49 & 36 \\
\hline Guainía & 0 & 0 & 1 & 1 & 1 & 26 & 1 & 1 & 0 & 0 & 0 & 1 & 1 & 1 & 1 \\
\hline Guaviare & 1 & 1 & 1 & 2 & 1 & 0 & 0 & 2 & 2 & 0 & 0 & 4 & 1 & 2 & 1 \\
\hline Huila & 12 & 0 & 4 & 0 & 10 & 26 & 3 & 12 & 46 & 19 & 33 & 82 & 68 & 30 & 18 \\
\hline Guajira & 0 & 2 & 5 & 3 & 14 & 22 & 6 & 17 & 27 & 8 & 27 & 24 & 15 & 10 & 4 \\
\hline Magdalena & 18 & 3 & 4 & 9 & 32 & 36 & 32 & 58 & 44 & 8 & 58 & 65 & 34 & 16 & 11 \\
\hline Meta & 15 & 0 & 15 & 2 & 13 & 16 & 15 & 18 & 24 & 12 & 37 & 42 & 10 & 14 & 29 \\
\hline Nariño & 22 & 0 & 7 & 1 & 21 & 11 & 6 & 13 & 30 & 17 & 67 & 37 & 68 & 30 & 33 \\
\hline Norte de Santander & 10 & 2 & 15 & 15 & 23 & 35 & 11 & 2 & 32 & 12 & 34 & 70 & 28 & 0 & 7 \\
\hline Putumayo & 12 & 7 & 0 & 2 & 5 & 2 & 3 & 6 & 6 & 11 & 5 & 4 & 47 & 38 & 23 \\
\hline Quindío & 2 & 2 & 0 & 2 & 2 & 4 & 16 & 3 & 13 & 6 & 15 & 43 & 15 & 27 & 5 \\
\hline Risaralda & 2 & 6 & 6 & 1 & 2 & 19 & 12 & 15 & 12 & 6 & 22 & 44 & 31 & 12 & 8 \\
\hline San Andrés y Prov. & 0 & 0 & 0 & 1 & 0 & 0 & 1 & 0 & 0 & 0 & 0 & 0 & 2 & 0 & 1 \\
\hline Santander & 19 & 10 & 20 & 33 & 21 & 44 & 49 & 29 & 55 & 47 & 56 & 90 & 19 & 28 & 21 \\
\hline Sucre & 13 & 2 & 6 & 11 & 17 & 15 & 19 & 28 & 23 & 7 & 28 & 36 & 36 & 25 & 12 \\
\hline Tolima & 19 & 11 & 14 & 9 & 13 & 30 & 16 & 26 & 83 & 17 & 61 & 55 & 60 & 62 & 19 \\
\hline Valle del Cauca & 27 & 7 & 6 & 8 & 4 & 21 & 37 & 54 & 75 & 24 & 67 & 122 & 78 & 59 & 26 \\
\hline Vaupés & 0 & 0 & 1 & 0 & 0 & 1 & 0 & 0 & 0 & 0 & 0 & 1 & 0 & 3 & 2 \\
\hline Vichada & 1 & 0 & 2 & 4 & 4 & 0 & 2 & 4 & 3 & 0 & 0 & 1 & 4 & 1 & 4 \\
\hline Total anual & 291 & 104 & 224 & 275 & 415 & 565 & 490 & 616 & 1086 & 439 & 1115 & 1587 & 1158 & 673 & 435 \\
\hline
\end{tabular}

Fuente: Vásquez, J. E., Gómez, M. I. \& Martínez, H. D. (2017) Gestión del riesgo de desastres, ordenamiento territorial, reasentamiento y reubicación: correlación desequilibrada para el ejercicio de derechos humanos desde retrospectivas jurídicas en Colombia. En: IX Seminario Internacional de Investigación en Urbanismo. Barcelona. 
De la sistematización de víctimas en la ocurrencia de eventos naturales se fortalece que la actuación del Estado ha sido antes que nada reactiva, dejando de aplicar medidas preventivas que salvarían muchas vidas y ahorrarían muchos recursos públicos. Para visibilizar dicha postura en materia de Gestión del Riesgo de Desastres, se puede hacer énfasis, a manera de ejemplo, en departamentos como Antioquia, Bolívar, Chocó, Cundinamarca, Santander y Tolima, los cuales frente al evento inundación presentan entre los años 2007 - 2012 un gran número de repeticiones que va aumentando anualmente, lo que contradice la finalidad preventiva de las normas en materia de gestión del riesgo, porque se considera que a mayor número de repeticiones del evento en determinado territorio, el Estado adquiere y especializa las potencialidades que le deberían proporcionar mayor y mejor capacidad de respuesta de manera preventiva a través de estudios de amenazas sobre éste que deriven en un diagnóstico que determine como podría ir evolucionando y sobreviniendo el fenómeno.

La capacidad preventiva del Estado se supone evolucionada y especializada si se tiene presente, por ejemplo, que desde el Decreto Ley 919 de 1989 se disponía la inclusión del componente de prevención de desastres en los planes de desarrollo de las entidades territoriales (artículo 6) para lo cual se contaba con la concurrencia de la Oficina Nacional para la Prevención y Atención de Desastres (artículo 59, 3, h) y se disponía la colaboración de las Corporaciones Autónomas Regionales (artículo 64, l) y la Ley 1523 de 2012 impone la integración de la gestión del riesgo en la planificación territorial y del desarrollo (artículo 39), la incorporación de la gestión del riesgo en la planificación (artículo 40) "la incorporación efectiva del riesgo de desastre como un determinante ambiental que debe ser considerado en los planes de desarrollo y de ordenamiento territorial, de tal forma que se aseguren las asignaciones y apropiaciones de fondos que sean indispensables para la ejecución de los programas y proyectos prioritarios de gestión del riesgo de desastres en cada unidad territorial." (artículo 41), y el análisis específico del riesgo y la formulación de planes de contingencia por parte de "entidades públicas o privadas encargadas de la prestación de servicios públicos, que ejecuten obras civiles mayores o que desarrollen actividades industriales o de otro tipo que puedan significar riesgo de desastre para la sociedad" (artículo 42). De ahí la norma contemple suficientes estrategias y disposiciones para que lo que se entre a evitar no sea propiamente la repetición del evento sino las consecuencias que éste puede ir generando a su paso en términos de las afectaciones a la población.

Pese a que la normativa proporciona todo un referente con función de prevención general, incluso departamentos como Antioquia y Cundinamarca que cuentan con una buena capacidad técnica y financiera para la ejecución de planes y proyectos sobre la gestión del riesgo de desastres, son los que más registros aportan al consolidado histórico nacional respecto de los eventos inundación, deslizamiento y avalancha. Por ello que ya en muchas oportunidades le haya correspondido al poder judicial asumir el conocimiento de las causas en las que se demanda el reconocimiento y protección de derechos amenazados y vulnerados desde la función de gestión del riesgo de desastres.

Algunos de los casos que han llegado a los estrados judiciales y que tienen estrecha relación con una de las problemáticas principales que aquí se ha descrito sobre los eventos naturales hidrometeorológicos, se pueden ver en pronunciamientos de la Corte Constitucional. Las sentencias T-199 de 2010, T-041 de 2011, T-355 de 2013 y T-648 de 2013 comparten en común el origen en eventos naturales relacionados con el efecto de los cambios atmosféricos en diferentes fuentes de agua, estos casos están estrechamente 
relacionados en la medida en que se está pidiendo el amparo fundamental de derechos como la vida, la salud, el debido proceso, la vivienda digna, entre muchos otros, por la no atención a comunidades que están asentadas en zonas de riesgo, producto de la probabilidad de ocurrencia de alguno de estos eventos naturales.

En los casos de las sentencias T-199 de 2010 y T-041 de 2011 se trató de situaciones en los que la comunidad (de los barrios Nuevo de Caracolí -Antioquia- y el Jardín del Quibdó -Chocó- respectivamente) pone de presente los riesgos causados o inminentes que las autoridades no han prevenido y que ha conllevado a la producción de inundaciones y deslizamientos que generan lesiones y peligro de muerte, además de los daños estructurales en las viviendas que pueden traer como consecuencia el desplazamiento de las personas que habitan estas zonas, porque no se realiza ningún tipo de actividad para mitigar los efectos que causa la lluvia en estas zonas del país. Los hechos específicos que generaron la sentencia de tutela 199 del año 2010 se pueden resumir de la siguiente manera:

Narran que en el terreno donde están construidas sus residencias se han presentados desprendimientos de rocas y deslizamientos de tierras que han afectado sus viviendas. Específicamente dan cuenta que en octubre de 2008 ocurrió el desprendimiento de una roca que afectó la vivienda de la Sra. María Encarnación Urrego y el 26 de abril de 2009 se deslizó la barranca junto con un árbol de matarratón, hecho que causó daños a algunos de los inmuebles antes identificados.

Afirman que desde el año 1998 han dirigido diversas peticiones a la administración municipal con la finalidad de que se adopten medidas para estabilizar los terrenos donde están construidas sus residencias, pero que hasta la fecha tales solicitudes han sido infructuosas.

[...] Afirman que en los inmuebles afectados habitan niños, adolescentes y personas de la tercera edad y que en la vivienda del Sr. Gonzalo Pineda y la Sra. Mariela Franco "funciona una guardería del ICBF". La vida e integridad de todas estas personas estaría en riesgo inminente debido a los deslizamientos. (Corte Constitucional, 2010).

Particularmente en las sentencias T-355 de 2013 y T-648 de 2013 se encuentra que, además de la afectación que sufren determinadas poblaciones por la ocurrencia de eventos naturales que les ocasionan múltiples daños a la integridad, a la salud y a la estructura de sus viviendas, también se ven afectados por el actuar negligente o intencional de las autoridades estatales en la atención preferente a determinadas personas por razones de corrupción y clientelismo y con el objetivo de garantizar votos para próximas elecciones. Esto se resalta de manera mucho más evidente en la sentencia T-648 porque se trató del riesgo de pobladores de diversos municipios de los departamentos de Bolívar, Sucre y César, en los que aproximadamente 3.800 personas presentaron acción de tutela porque se consideraron afectadas no solo por el fenómeno natural de la inundación, sino que además no fueron beneficiarias de los respectivos subsidios y beneficios que se habían concedido a otras personas de manera amañada:

Los accionantes manifiestan que residen en los Municipios de Córdoba, San Jacinto del Cauca y Margarita -Mompox (Departamento de Bolívar), Majagual y San Marcos (Departamento de Sucre), La Gloria (Departamento de Cesar) y Pedraza (Departamento de Magdalena); los cuales hicieron parte de las zonas más afectadas del país por el fenómeno de la niña ocurrido en los meses de septiembre a diciembre de 2011. 
Informaron que los Comités Locales para la Prevención y Atención de Emergencias y Desastres CLOPAD realizaron un censo para determinar el número de familias que serían beneficiarias de la asistencia económica anunciada por el Presidente Juan Manuel Santos. Pese a estar incluidos en el censo y a encontrarse en igualdad de condiciones con otras familias que ya fueron beneficiarias de la ayuda, aseguran que no han recibido el beneficio.

Afirmaron que, en el trámite para obtener el beneficio, las autoridades municipales incurrieron en graves irregularidades que impidieron la entrega de la ayuda económica. En consecuencia, solicitan que se le ordene a la Unidad Nacional para la Gestión del Riesgo de Desastres incluirlos en las listas de damnificados, con la finalidad de obtener el beneficio equivalente a un millón quinientos mil pesos ( $\$ 1^{\prime} 500.000$ ) y se autorice su entrega en el menor tiempo posible. (Corte Constitucional, 2013)

\section{Conclusiones}

La regularización de la gestión del riesgo del desastre es un tema que se ha dado lugar en el ámbito internacional y consecuentemente en el nacional desde la década de los años setenta, por lo que Colombia es un Estado que desde entonces viene definiendo un marco normativo e institucional que regula la materia. Eso hace del caso colombiano una experiencia que frente a la gestión del riesgo de desastres ha convocado la acción legislativa, reglamentaria, política y judicial sin que se trate de un tema nuevo para el país. La década de los años setenta representa el inicio de un recorrido normativo que no solo se amplía y articula con el escenario internacional, sino que eleva a rango constitucional muchas de las dimensiones propias de la prevención de los desastres técnicamente previsibles para las autoridades estatales, al punto de reconocer positivamente dicha categoría como un derecho colectivo que demanda el cumplimiento de obligaciones prestacionales de parte del Estado en el ejercicio de su función administrativa, descentralizada territorialmente en las autoridades integradas en el SNGRD.

De la descripción de la reconstrucción normativa e institucional se colige que dentro del fragmento cronológico relacionado con el periodo comprendido entre el Decreto 1355 de 1970 y la Ley 46 de 1988 se presentaron variaciones importantes que progresivamente fueron desarrollando un sistema de prevención y atención de desastres. Es de resaltar que en ese lapso de tiempo existieron figuras que, una vez creadas, se mantuvieron vigentes con leves o inclusive sin ninguna modificación pese al trascurso del tiempo, al cambio de las condiciones sociales y económicas y al cambio de normatividad que ello implicaba, tal es el caso del Fondo Nacional de Calamidades (hoy Fondo Nacional para la Gestión del Riesgo de Desastres), del análisis de vulnerabilidad, de la inclusión del sector privado en la materia y del sistema de información como mecanismo fundamental para la identificación del riesgo en el país.

En el tercer periodo vale destacar como el ordenamiento jurídico colombiano dotó la gestión del riesgo de desastres con la Red Nacional de Centros de Reserva para la atención de emergencias, modificada y actualizada a través del Decreto 3696 de 2009; la incorporación del Sistema de Bomberos al Sistema Nacional de Prevención y Atención del Riesgo de Desastres, y la incorporación de la gestión del riesgo en el plan de ordenamiento territorial, principal instrumento de planeación físico territorial a cargo de los municipios y distritos en Colombia, recientemente determinado por el Decreto 1807 de 2014 que reglamenta la incorporación de los eventos naturales de inundaciones, avenidas torrenciales u movimientos en masa en los procesos de revisión y actualización del citado instrumento normativo. 
La condición de vulnerabilidad del país se encuentra enmarcada en la no concreción de políticas efectivas que deriven en una fuerte y eficaz atención del riesgo, ya que al tenor de diferentes posturas que devienen tanto de la esfera gubernamental como de ámbitos técnicos, el país aún continúa con una tendencia de suerte "reactiva" y no preventiva y planeadora, es decir, solo reacciona cuando ya el evento es inminente o se ha presentado a pesar de su posible previsibilidad o constancia en determinados departamentos, incluso a pesar de la intensidad y reiteración de los mismos. Mientras no se pase de una actitud reactiva a una realmente preventiva el país seguirá en condiciones de vulnerabilidad frente a los trece (13) eventos mencionados y a los nuevos riesgos a los que se tenga que enfrentar producto del cambio climático y la degradación ambiental.

Desde las diversas figuras que ofrece el ordenamiento territorial colombiano se hace posible prevenir los desastres desde una objetiva y real identificación de las amenazas, como por ejemplo desde la manera en que utiliza adecuadamente el territorio de acuerdo a la vocación que tenga, de allí que herramientas tales como los planes de manejo y ordenación de cuencas y los planes de ordenamiento territorial sean elementos fundamentales a la hora de la gestión del riesgo de desastre.

\section{Bibliografía}

Arango, R. (2005) El concepto de derechos sociales fundamentales. Bogotá: Editorial Legis.

Asamblea General de la Organización de las Naciones Unidas (1991) Resolución 46/182, por medio de la cual se aprueba el fortalecimiento de la coordinación de la asistencia humanitaria de emergencia del sistema de las Naciones Unidas. Nueva York: Asamblea General de la Organización de las Naciones Unidas.

Asamblea Nacional Constituyente. Constitución Política de Colombia (1991). Versión digital disponible en: http://www.alcaldiabogota.gov.co/sisjur/normas/Norma1. isp?i=4125

Cardona, O. D. (2005) La gestión del riesgo colectivo. Recuperado de file:///C:/Users/ usuario/Downloads/Gestion Riesgo Ciudad Laboratorio21-09-05LaRED\%20(1).pdf

Conferencia Mundial sobre la Reducción de Desastres (2005) Informe de la Conferencia Mundial sobre la Reducción de los Desastres. A/CONF.206.6. Hyogo: Organización de las Naciones Unidas. Conferencia Mundial sobre la Reducción de los Desastres Naturales (1994) Informe de la Conferencia Mundial sobre la Reducción de los Desastres Naturales. A/CONF.172/9. Yokohama: Organización de las Naciones Unidas.

Congreso de la República de Colombia (1979). Ley 9 de 1979. Versión digital disponible en: http://www.alcaldiabogota.gov.co/sisjur/normas/Norma1.jsp?i=1177.

Congreso de la República de Colombia (1996) Ley 322 de 1996. Versión digital disponible en: http://www.alcaldiabogota.gov.co/sisjur/normas/Norma1.jsp?i=350

Congreso de la República de Colombia (1997) Ley 388 de 1997. Versión digital disponible en: http://www.alcaldiabogota.gov.co/sisjur/normas/Norma1.jsp?i=339.

Congreso de la República de Colombia (1998) Ley 46 de 1998. 
Congreso de la República de Colombia (2012) Ley 1523 de 2012. Versión digital disponible en: http://www.alcaldiabogota.gov.co/sisjur/normas/Norma1. isp?i=47141

Congreso de la República de Colombia (2012) Ley 1575 de 2012. Versión digital disponible en: http://www.alcaldiabogota.gov.co/sisjur/normas/Norma1. jsp?i=48943.

Corte Constitucional de la República de Colombia (2010) Sentencia T - 199.

Corte Constitucional de la República de Colombia (2011) Sentencia T - 041.

Corte Constitucional de la República de Colombia (2013) Sentencia T - 355.

Corte Constitucional de la República de Colombia (2013) Sentencia T - 648.

Cumbre Mundial sobre Desarrollo Sustentable (2002) Declaración de Johannesburgo sobre Desarrollo Sustentable. Johannesburgo: Organización de las Naciones Unidas.

Gobierno de la República de Colombia (1989) Decreto Ley 919.

López, J. (2009). La garantía de los Derechos Sociales. Bogotá, Pontificia Universidad Javeriana - Ediciones Gustavo Ibañez.

Ministerio de Desarrollo (1984). Decreto 1547 de 1984. "Por medio del cual se crea el Fondo Nacional de Calamidades y se dictan normas para su organización y funcionamiento". Versión digital disponible en: http://www.icbf.gov.co/cargues/avance/ docs/decreto 1547 1984.htm.

Ministerio de Interior y de Justicia de la República de Colombia. (2009) Decreto 3696 de 2009. "Por medio del cual

Ministerio de Justicia (1970). Decreto 1355 de 1970. "Por medio del cual se dictan normas sobre policía". Versión digital disponible en: http://www.alcaldiabogota. gov.co/sisjur/normas/Norma1.jsp?i=6945.

Ministerio del Interior de la República de Colombia (1995) Decreto 2190 de 1995. "Por medio del cual se ordena la elaboración y desarrollo del Plan Nacional de Contingencia contra derrames de Hidrocarburos, Derivados y Sustancias Nocivas en aguas marinas, fluviales y lacustres". Versión digital disponible en: $\underline{\text { http:// }}$ www.minambiente.gov.co/images/BosquesBiodiversidadyServiciosEcosistemi$\mathrm{cos} / \mathrm{pdf} /$ Normativa/Decretos/dec 2190_141295.pdf.

Ministerio de Vivienda, Ciudad y Territorio (2014) Decreto 1807 de 2014. "Por medio del cual se reglamenta el artículo 189 del decreto Ley 019 de 2012 en lo relativo a la incorporación de la gestión del riesgo en los planes de ordenamiento territorial y se dictan otras disposiciones". Versión digital disponible en: http:// portal.gestiondelriesgo.gov.co/Documents/SRR/decrerto_1807_19_\%20septiembre 2014.pdf 
Ministerio de Vivienda, Ciudad y Territorio (2015) Decreto 1077 de 2015. "Por medio del cual se expide el Decreto Único Reglamentario del Sector Vivienda, Ciudad y Territorio." Versión digital disponible en: http://www.minvivienda.gov.co/Normativalnstitucional/1077\%20-\%202015.pdf.

Presidencia de la República de Colombia (1995) Decreto 969 de 1995. "Por medio del cual se organiza y reglamenta la red nacional de centros de reserva para la atención de emergencias". Versión digital disponible en: http://www.alcaldiabogota.gov.co/sisjur/normas/Norma1.jsp?i=4697.

Presidencia de la República de Colombia (1998) Decreto 93 de 1998. “Por medio del cual por el cual se adopta el Plan Nacional para la Prevención y Atención de Desastres". Versión digital disponible en: http://www.alcaldiabogota.gov.co/sisjur/ normas/Norma1.jsp?i=3454.

Presidencia de la República de Colombia (2012) Decreto Ley 019 de 2012. "Por medio de la cual se dictan normas para suprimir o reformar regulaciones, procedimientos y trámites innecesarios existentes en la Administración Pública". Versión digital disponible en: http://www.alcaldiabogota.gov.co/sisjur/normas/ Norma1.jsp?i=45322.

Vásquez Santamaría, J. E., Gómez Vélez, M. I. \& Martínez Hincapié, H. D. (2017) "Gestión del riesgo de desastres, ordenamiento territorial, reasentamiento y reubicación: correlación desequilibrada para el ejercicio de derechos humanos desde retrospectivas jurídicas en Colombia." En: IX Seminario Internacional de Investigación en Urbanismo. Barcelona. Disponible On Line en: https://arqdis.uniandes.edu.co/Seminario SIIU/memorias.html 